\title{
Requiem for the grand round
}

\author{
Authors: Maham Stanyon ${ }^{A}$ and Shahid Anis Khan ${ }^{B}$
}

The time-honoured tradition of Grand Round is firmly rooted in medical education, but has little evidence for its effectiveness or its impact on patient management. A mode of didactic teaching, Grand Round has lost its appeal in modern medical education with dwindling attendance at Grand Rounds worldwide. Once a platform for eminence-based medicine and a cross fertilisation of medical ideas, emphasis on subspecialisation and clinical governance, combined with rota, trainee engagement and attendance failures has made Grand Round obsolete. To survive, Grand Round must have evidence for its effectiveness in medical education. It must be able to compete with other forms of teaching and adapt by using technology to reach trainees. Engaging the audience and encouraging participation needs to be woven into the fabric of the modern Grand Round, alongside learning clinical skills and developing clinical reasoning. Understanding the needs of today's trainees and their involvement in formulating the Grand Round programme is vital. Attendance at Grand Round is a recognised measure of its success and will be used in its evaluation. Questions still remain regarding the future of Grand Round. Its survival will depend upon its ability to change with time and reclaim its place as a credible tool to promote learning.

KEYWORDS: Grand Round, education

The time-honoured practice of Grand Round is a familiar feature of hospital life. Steeped in formality and tradition, older than the health service, Grand Round is a component of most UK hospital teaching and established practice around the globe. ${ }^{1-3}$ Competing in today's dynamic educational arena, there are mounting concerns that the Grand Round is losing its appeal among the medical faculty, ${ }^{4-7}$ a dinosaur in danger of being declared an outdated mode of delivering medical education.

\section{History of the Grand Round}

Historically the practice of Grand Round dates back several centuries, but its exact origins are unknown. Early Grand

Authors: ${ }^{A}$ academic GP ST1, Imperial GP Speciality Training, Department of Primary Care and Public Health, Imperial College London, London, UK; ${ }^{B}$ director of Medical Education East and North Hertfordshire NHS Trust, Lister Education Centre, Lister Hospital, Stevenage, UK
Rounds consisted of an oral presentation where speakers were invited for their expertise, educating colleagues and eager trainees in crowded auditoriums with 'in the flesh' patient examples, while demonstrating outstanding presentational skill. ${ }^{8}$ Over time, with improvement in diagnostic aids and an emphasis on confidentiality, patients were gradually replaced by electronic presentation tools. Focus from clinical signs shifted to investigations, procedures and surgical techniques. In addition, a shift towards evidence-based practice turned some Grand Rounds into lectures on research and statistical analysis, thus leading some to question their contribution to basic medical education. ${ }^{9}$

\section{The impact of the Grand Round}

The impact of the Grand Round on doctors' clinical management is difficult to evaluate, with no clear evidence base to support its continuation. ${ }^{10}$ So why has it weathered centuries? One hypothesis explaining the longevity of the Grand Round is the limited availability of literature to the medical workforce which dominated practice until recent times. Grand Round was not only a source of information and a medium to keep abreast with technology, but also a stage where eminent scholars mesmerised their audience with the brilliance of their skills. It was a platform where most specialities interacted on a regular basis with cross fertilisation of ideas, generation of debate and integration of workforce. ${ }^{12}$ This complemented the emphasis on general training rather than organ-based sub-specialisation which predominates today.

\section{Evolution of the Grand Round}

Grand Round evolved over time into a largely lecture-based, less interactive model, which some have deemed an expensive way of delivering medical training. ${ }^{7}$ Others have highlighted a lack of curricular structure in topic selection and a paucity of evaluation for learning gains. ${ }^{11}$ A further feature signalling the demise of the Grand Round is the dwindling attendance which led others to look at factors responsible and suggested examining the needs of trainees. ${ }^{3}$

In the era of information overload and availability of upto-date information at the fingertips of most doctors, the role of the Grand Round in medical education seems blurred. Emphasis on sub-specialisation and focus on single organ speciality makes it difficult to engage super specialists in general topics of medical interest, the hallmark of Grand Round. 


\section{Fall of the Grand Round}

The mêlée of training versus service needs, working time directive and changes to the shift system have taken their toll on Grand Round attendance. Changing the horizon of medical education, with the introduction of shorter training programmes, curriculum-based training, mandatory training and the development of core skills within a tight time frame, has left the Grand Round out of focus. At the other end of the spectrum, changes to the consultant contract, including the emphasis on programmed activities, clinics and operating sessions, have encroached upon the time previously earmarked for Grand Round. The reduced consultant presence at the Grand Round may have impacted adversely upon junior doctors' preference to attend.

For hospital management, the Grand Round is not just an educational resource but also a platform for dissemination of information to a large cohort of doctors. Mandatory training and clinical governance initiatives could be launched from this platform with perceived 'guaranteed attendance' of many consultants and trainees. Although the Grand Round can be used in this respect, overemphasis on governance may have discouraged some from attending.

\section{Future of the Grand Round}

For the Grand Round to survive threatened extinction and retain its position in medical education it will need to change. Working in partnership with trainees and encouraging regular feedback could shape future programmes. ${ }^{12}$ Trainees are focused on individual curriculum, and learning opportunities outside their curriculum are not being fully availed. It is strongly felt that Grand Round should be part of training ${ }^{13}$ and rostered into shifts for trainees. Furthermore, prominent in a Grand Round 'wish list' is that it should cater for the needs of all grades of doctors, with use of new technology to promote access to those less able to attend due to geographical constraints. Last but not least, evidence of attendance at Grand Rounds should be reviewed at the Annual Review of Competence Progression.

The Grand Round evolved early in the history of modern medical education and is internationally recognised as a tool for training and updating the medical workforce. Despite the differing opinions regarding its benefits, it provides a locally assessable continuing medical education programme for all grades of doctors. Its benefits of interaction among specialties should not be ignored. There is need for more audience participation, presentations with a focus on clinical problem solving, multi-professional interaction and trainee participation in its development. Research is needed to critically analyse this deeply engrained resource for its educational value and impact on patient care. It may not yet have been laid to rest, but failure to act now may hasten its demise and then we shall truly require a requiem for the Grand Round.

\section{References}

1 Richmond DE. The educational value of grand rounds. N Zeal Med J 1985;98:280-2.

2 McLeod PJ. Medical grand rounds: alive and well and living in Canada. Can Med Assoc J 1990;142:1053-6.

3 Tarala R, Vickery AW. Hospital grand rounds in Australia. Med J Aust 2005;19:592-4.

4 Kassirer JP, Kopelman RI. Clinical problem-solving at grand rounds. Hosp Pract 1990;25:51-9.

5 Parrino TA, White AT. Grand rounds revisited: results of a survey of U.S. departments of medicine. Amt J Med 1990;89:491-5.

6 Inglefinger FJ. The graying of grand rounds. N Engl J Med 1978;299:772.

7 Hebert RS, Wright SM. Re-examining the value of medical grand rounds. Acad Med 2003;78:1248-52.

8 Salman RAS, Stone J, Warlow C. What do patients think about appearing in neurology "grand rounds"? J Neurol Neurosurg Psychiatry 2007;78:454-6.

9 Hebert RS, Wright SM. Re-examining the value of medical grand rounds. Acad Med 2003;78:1248-52.

10 Davis DA, Thompson MA, Oxman AD Haynes H. Evidence for the effectiveness of CME. A review of 50 randomized controlled trials. JAMA 1992;268:1111-7.

11 Lewkonia RM, Murray FR. Grand rounds: A paradox in medical education. CMAJ 1995;152:371-6.

12 Dolcourt JL, Zuckerman G, Warner K. Learners' decisions for attending Pediatric Grand Rounds: a qualitative and quantitative study. BMC Med Educ 2006;6:26.

13 Mueller PS, Litin SC, Sowden ML, Habermann TM, LaRusso NF. Strategies for improving attendance at medical grand rounds at an academic medical centre. Mayo Clin Proc 2003;78:549-53.

Address for correspondence: Dr SA Khan, East and North Hertfordshire NHS Trust, Lister Education Centre, Lister Hospital, Corey's Mill Lane, Stevenage SG1 4AB, UK. Email: shahidkhan@nhs.net 